\title{
SALARIO EMOCIONAL: UNA SOLUCIÓN ALTERNATIVA PARA LA MEJORA DEL RENDIMIENTO LABORAL.,2
}

Emotional salary: an alternative solution for labor performance improvement

Fecha de recepción: 19 de febrero de 2020 Fecha de aceptación: 5 de junio de 2020

1- Ana Karen Espinoza Saldívar. Grado académico: Estudiante de la Maestría en Administración. Adscripción: Universidad Autónoma de Ciudad Juárez. Correo electrónico: kareen.e@hotmail.com. ORCID ID: https://orcid.org/0000-0002-01414438

2- Juan Alfonso Toscano Moctezuma. Grado académico: Doctorado en Ciencias de la Administración. Adscripción: Universidad Autónoma de Ciudad Juárez. Correo electrónico: jtoscano@uacj.mx. ORCID ID: https://orcid.org/0000-00028543-3600 


\section{RESUMEN}

Múltiples beneficios reflejados en el incremento del rendimiento, motivación y satisfacción laboral de los trabajadores, según diversos estudios, están fuertemente ligados a las retribuciones de carácter no económico, mejor conocidas como "salario emocional". Por tal motivo, la presente investigación tiene como objetivo presentar los resultados de la revisión literaria a nivel internacional sobre los beneficios de su implementación. Los resultados evidencian el incremento del rendimiento, productividad, proactividad, eficiencia y compromiso de los colaboradores en pro del logro de los objetivos de la organización por el uso de dicho incentivo.

Palabras claves: Salario Emocional, Rendimiento Laboral, Productividad, Compromiso.

\section{ABSTRACT}

According to diverse studies, reflected multiple benefits in performance, motivation and job satisfaction increasing by the organization employees are strongly linked to a non-economic remuneration, better known as "emotional salary". For this purpose, this research aims to present the results of an international sources literature review on the benefits of its implementation. Results show performance, productivity, proactivity, efficiency and engagement of employees increasing towards the objectives achievement of the organization using that incentive.

Keywords: Emotional Salary, Labor Performance, Productivity, Engagement.

Jel: J32, J33 


\section{Introducción}

$\mathrm{E}$ 1 presente artículo plantea de qué manera el rendimiento, motivación, satisfacción y productividad del empleado se ve mejorado bajo el uso de un esquema de retribuciones mayormente conocido como "salario emocional", el cual se define como cualquier tipo de compensación intangible que el trabajador percibe a cambio de sus servicios (Baguer, 2005, citado por Augusto, Campagnolli, Canuto, y Graziano, 2018).

La investigación surge a raíz de conocer más a fondo que tipo de beneficios trae consigo el uso salario emocional dentro de una compañía, ya que según los autores Buqueras y Cagigas (2017) los trabajadores más satisfechos son los más motivados, en consecuencia, los más productivos. Dicha satisfacción se logra no sólo a través de un buen sueldo, sino también por medio de un equilibrio entre la vida personal, laboral y familiar.

Cabe resaltar que, a pesar de aparentemente reflejar resultados positivos, compañías nacionales e internacionales aún se encuentran renuentes a la implementación del mismo, debido a que lo consideran como un gasto, que visto desde el punto de vista contable, lo es, ya que se necesita inversión si es que la compañía opta por ofrecer planes de carrera, adaptar una zona como área de descanso, renovar el mobiliario u ofrecer tarjetas de regalo o membresías, y aun así, no tener la certeza de que con esos cambios el personal asegure su permanencia y engagement.

Después de esta breve introducción, es menester aclarar que en la literatura revisada se encontraron pocos estudios sobre los beneficios del uso del salario emocional en México, por ello, se identificó la necesidad de indagar y realizar una revisión literaria a nivel internacional, debido a que hoy en día son factores importantes de uso organizacional que reflejan mejoras continuas dentro de las organizaciones.

De manera que el presente documento se estructura de la siguiente manera: posterior al apartado de la introducción, se describe de manera resumida la información referente a los beneficios de las retribuciones, en la segunda sección se encuentra la metodología empleada para la realización del presente artículo. Seguidamente, se desglosan de manera más precisa los resultados de la búsqueda documental. Finalmente, para concluir, se plantean algunas conclusiones derivadas del documento.

\section{Revisión de literatura}

Dentro de la literatura consultada se encontraron varios puntos de vista respecto al salario emocional, los cuales a continuación se muestran:

Desde hace tiempo, se han redactado artículos, libros, se han realizado conferencias y talleres sobre cómo conseguir que un empleado haga lo que el jefe quiera o le pida; siendo todo lo referente a la motivación laboral un tema complejo y lo que se ha logrado encontrar a base de investigaciones, es de baja certeza. El artículo de 1969, reimpreso en 2003 por Harvard Business Review sobre las teorías de Frederick Herzberg, no pretende mostrar remedios milagrosos para lograr una motivación en el empleado, sin embargo, las ideas que se expresan dentro del artículo han sido probadas por corporaciones y organizaciones, por lo tanto, se espera que sean de ayuda (Herzberg, 1969). 
Respecto a qué beneficios tiene el mantener al empleado satisfecho, los autores, Augusto et al., (2018), señalan en su artículo que los empleados satisfechos tienden a ser más productivos, son quienes se encuentran dentro de un puesto en áreas de liderazgo y perciben mejores ingresos. Sin embargo, la felicidad del empleado no es un beneficio exclusivo de él, sino también de la compañía y su éxito organizacional; si el trabajador labora con un nivel de motivación alto, trabajará de manera más armónica. Por ello, se dice que la aplicación correcta del salario emocional es altamente efectiva debido a los factores que los componen, como el reconocimiento, la retroalimentación, el desarrollo profesional y el interés por las necesidades del trabajador.

Un enfoque interesante y de interés nacional sobre el salario emocional, se encuentra dentro del artículo de los autores López, Chávez, Peña y Guevara (2018) quienes realizaron su investigación en Coahuila, dentro del cual se confirmó la existencia entre los niveles de satisfacción del empleado al percibir un alto nivel de participación activa dentro de la organización.

Ahora, ¿Cómo se puede compensar al trabajador más allá de lo económico?, ¿Es posible lograr el equilibrio laboral y personal del empleado, y al mismo tiempo incrementar la competitividad de la empresa? El autor Puyal (2016) dentro de su artículo: “El salario emocional, clave para reducir el estrés", menciona que el salario emocional es un instrumento facilitador para la conciliación entre la vida laboral y personal del empleado.

Ya que según la autora Granados (2011) la vida laboral del empleado se ve afectada, no sólo por factores intrínsecos que se presentan en el entorno del trabajo, también por todo lo acontece fuera de, lo extra laboral, factores que se integran en la cotidianidad del empleado como la familia, amigos, red de contactos, ocio y el tiempo libre. Esta interdependencia da lugar a la relación estrecha entre la vida laboral y personal (o extra laboral).

En cuanto a la flexibilidad laboral se dice que, la presencia no es lo mismo que el rendimiento, la valoración de un empleado se mide a través de su rendimiento y del valor que este mismo aporte al negocio, no en las horas de presencia en la oficina, esto según Cervantes (2005), director de recursos humanos de IBM España y Portugal, en su artículo "Las ventajas de la empresa flexible".

A través de su libro "Dejemos de perder el tiempo: Los beneficios de optimizar los horarios", los autores Buqueras y Cagigas (2017) explican de manera amplia los beneficios de la correcta gestión del tiempo, señalando que una empresa comprometida con la excelencia en la gestión de tiempo obtiene ventajas competitivas como: mayor productividad y rendimiento al máximo, asimismo, para el empleado se presenta una conciliación de la vida personal y laboral y la empresa define su responsabilidad y respeto hacia el empleado.

Por su parte, Restrepo (2014), dentro de su articulo realizado en Colombia, enlista los elementos implicitos en las jornadas flexibles más valorados por los trabajadores, entre los que se encuentran: el teletrabajo, la semana reducida, y el horario de entrada y salida flexibles. La autora menciona que dicha flexibilidad mejora la motivación, concentración y rendimiento del empleado, por ende, facilitará la productividad y la rentabilidad. Además apunta que el salario emocional convierte a una empresa en un "lugar bueno para trabajar".

En la actualidad la demanda laboral para cargos especializados ha superado la oferta, lo que provoca una guerra de talentos y que la retención del personal se vuelva un desafío. Gonzales (2009) indagó sobre la efectividad y los alcances de las estrategias de retención del personal en 89 organizaciones productivas 
de Antioquia, Colombia, los resultados se encuentran a lo largo de este documento. Su investigación fue realizada entre 2008 y 2009.

Casi por concluir, destacaremos que las investigaciones realizadas tanto en España como en Chile por Rocco (2009), Nicolás (2011) y Martín (2017), quienes convergen en que la satisfacción laboral trae consigo beneficios muy grandes para la organización. Rocco (2009) realizó su investigación con la intensión de crear una reflexión de la relación del salario emocional y satisfacción laboral en un escenario económico. De forma similar, Martín (2017) buscó saber qué factores satisfacen al empleado para poder incrementar la productividad de este y, por último, Nicolás (2011) busca dar respuesta a la pregunta que todos los gestores de las empresas se hacen, ¿Por qué la empresa no logra que los empleados den más de sí mismos y se comprometan con la organización?

A manera de síntesis de este apartado, en la Tabla 1. Revisión de la Literatura, se encuentran los resultados obtenidos por los investigadores:

Tabla 1. Revisión de la Literatura

\begin{tabular}{|c|c|c|}
\hline AUTORES & VARIABLE & RESULTADOS \\
\hline $\begin{array}{l}\text { Buqueras \& Cagigas (2017) } \\
\text { Restrepo (2014) }\end{array}$ & $\begin{array}{l}\text { Flexibilidad } \\
\text { laboral }\end{array}$ & $\begin{array}{l}\text { El otorgar flexibilidad laboral mejora la motivación, } \\
\text { concentración y rendimiento del empleado. }\end{array}$ \\
\hline $\begin{array}{c}\text { Rocco (2009) } \\
\text { Nicolás (2011) } \\
\text { Martín (2017) } \\
\text { Augusto et al. (2018) }\end{array}$ & $\begin{array}{l}\text { Satisfacción } \\
\text { laboral }\end{array}$ & $\begin{array}{l}\text { Un empleado satisfecho tiende a mejorar en ámbitos } \\
\text { como: productividad, creatividad, eficiencia, además } \\
\text { el sentido de pertenencia con la organización y el } \\
\text { compromiso hacia sus funciones y responsabilidades } \\
\text { se verá incrementado de manera positiva, reflejan- } \\
\text { do un impacto positivo interna y externamente a la } \\
\text { compañía. }\end{array}$ \\
\hline $\begin{array}{c}\text { Augusto et al. (2018) } \\
\text { Restrepo (2014) } \\
\text { Puyal (2006) }\end{array}$ & $\begin{array}{l}\text { Salario emo- } \\
\text { cional }\end{array}$ & $\begin{array}{l}\text { El uso correcto del salario emocional catapulta a la } \\
\text { empresa como un lugar bueno para trabajar, el em- } \\
\text { pleado lo valora debido al reconocimiento, la retroali- } \\
\text { mentación y desarrollo profesional percibido, además, } \\
\text { es un instrumento facilitador para la conciliación } \\
\text { entre la vida laboral y personal del empleado. }\end{array}$ \\
\hline
\end{tabular}

Fuente: Elaboración propia.

\section{Metodología}

Para la realización de esta revisión literaria en primer lugar, se consultaron diversas bases de datos que permitieron la obtención de la información a través de artículos de investigación académica relacionados con las variables a estudiar, entre ellas destacan: 
- Red de revistas científicas REDALYC

- Biblioteca Científica Electrónica en Línea SciELO

- Motor de búsqueda multidisciplinario Bielefeld Academic Search Engine BASE

- Buscador científico-académico Google Scholar

Posteriormente se clasificaron los estudios en base a las variables a estudiar, tales como: salario emocional, rendimiento laboral, satisfacción laboral, productividad, factores emocionales, etc. Luego se escogieron únicamente los que tenían mayor relevancia y objetivos similares a los de esta revisión documental.

Finalmente, el criterio utilizado para la estructura y redacción del presente artículo, fue el agrupar los artículos por semejanzas y convergencias.

\section{Resultados}

Cuando se ha concluido la etapa de formación profesional y nos adentramos al mundo laboral consiguiendo un empleo por vocación o simplemente porque llegó una oportunidad, éste se realiza a cambio de una retribución económica. Sin embargo, los ciclos de recesión que se han presentado recientemente, han dejado un temor en las empresas llevándolas a ser más conservadoras con sus presupuestos y cuidar las contrataciones, derivando con ello sueldos bajos con bajas posibilidades de aumentos, lo que genera descontento, altos niveles de rotación y baja productividad (Aranguren, 2020).

Lo anterior refleja claramente la situación actual por la que varias empresas están atravesando debido a la pandemia provocada por el virus COVID-19. A lo largo de estos meses se han presentado despidos masivos, bajas temporales, reducción de personal, incluso se ha presenciado el cierre definitivo de muchas empresas, lo que ha generado el descontento del empleado, ello reflejado a través de las diferentes plataformas de comunicación como noticieros, notas periodísticas, incluso por medio de las redes sociales.

Por fortuna, hoy en día el trabajador valora más otras variables que van más allá de un sueldo económico, como la flexibilidad de horarios, capacitaciones, beneficios sociales, clima organizacional, etc. En pocas palabras, el bienestar laboral. En este contexto, se sitúa la estrategia conocida como "salario emocional", es decir, aquel incentivo tangible o intangible que al ofrecerlo no representa gran costo para la empresa. Lo que refleja un ganar-ganar, puesto que el empleado mejorará su motivación y compromiso, afectando de forma directa su productividad, mientras la empresa retiene al talento y obtiene mejores resultados para su negocio (Aranguren, 2020).

De manera más a grosso y para ir conectando todas las ideas e información que se verá a lo largo de este artículo, a continuación, se explican los componentes de la definición de salario emocional:

La definición genética de salario emocional se compone del término "salario" porque se busca la identificación y la relación con contraprestación y también con reciprocidad, equidad y frecuencia. Se califica de "emocional" porque con independencia del satisfactor qué se trate (vacaciones, atención a necesidades personales, elementos satisfactores como en el contexto del clima laboral) busca aportar satisfacción a alguno de los motivos intrínsecos y muy en especialmente a los motivos trascendentes (Puyal, 2016, p. 45). 
El autor menciona que el salario emocional debe contener satisfacción, tanto en calidad como en cantidad, por ello, no hay que olvidar que cada uno de los empleados cuenta con necesidades distintas y cambiantes. Los incentivos emocionales mal aplicados pierden su eficiencia con el tiempo. Por dicha razón, el salario emocional es poco generalizable pero altamente eficaz y eficiente si se singulariza al asalariado (Puyal, 2016).

El punto anterior claramente se torna hacia una desventaja de la implementación de este, puesto que son pocas las empresas que pueden adecuar los incentivos a todas y cada una de las necesidades de sus empleados. Respecto a la información anterior, salario emocional se complementa con el siguiente concepto:

Salario emocional son todas aquellas retribuciones no económicas que un trabajador percibe con el fin de contribuir a que pueda satisfacer sus necesidades personales y familiares. Se articula a través de su carácter no monetario y de su efecto simbólico en calidad de vida y la productividad de las personas. Es decir, su naturaleza versa en que el carácter de lo que se intercambia busca compaginar las expectativas de las personas, articulando sus necesidades en diferentes dimensiones de su vida cotidiana, para, de esa manera, contribuir a un mayor equilibrio (González y De Avice, 2017, p. 80).

Por ello se dice que, el salario emocional no tiene otro fin más que construir una vida más saludable, productiva y significativa para los trabajadores y lograr así que compaginen su vida laboral y personal. Y a modo de conclusión, la autora comenta que la paga del salario emocional lo utilizan aquellas empresas que buscan incrementar sus estándares de calidad y productividad, y al a vez procuran disminuir los índices de ausentismo y rotación.

De lo anterior resulta necesario decir que, la asociación civil mexicana Asociación de Internet MX (2018) en su estudio "Búsqueda de empleo", enlistó los factores clave que un mexicano considera esencial a la hora de una toma de decisión laboral, o bien, elementos que puedan garantizar su permanencia en la compañía. A continuación, se muestran las tablas referidas.

Figura 1. Búsqueda de empleo

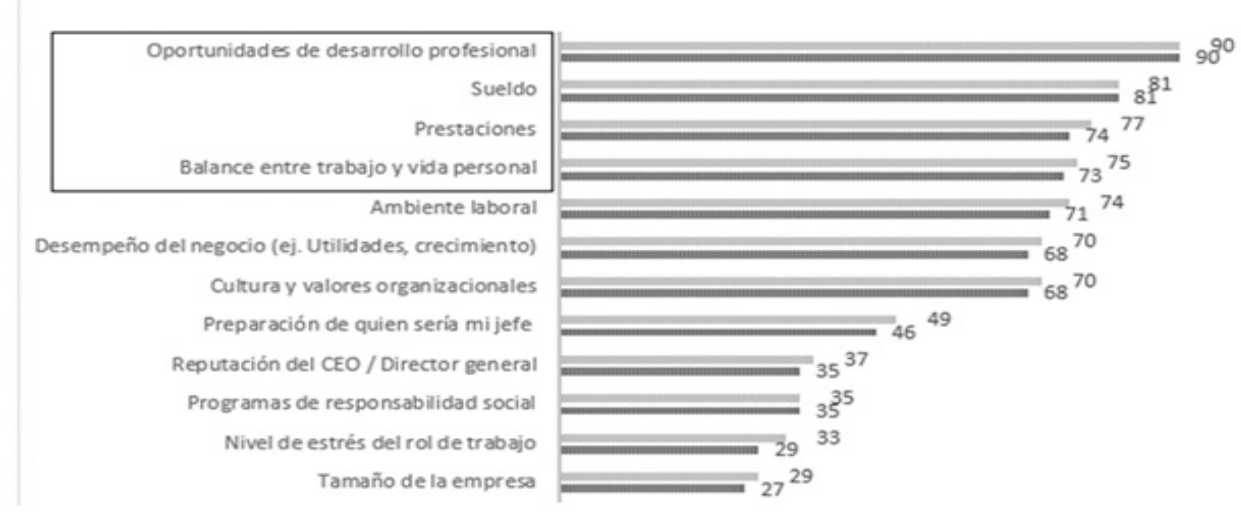

En esta figura se muestra la representación gráfica de los datos arrojados del estudio "búsqueda de empleo 2018" que, en comparación a los resultados obtenidos en el año 2017, los internautas encuestados dieron mayor importancia al salario emocional que las empresas puedan ofrecerles.

Fuente: Asociación de Internet MX (2018). 
Como bien muestra la figura, el empleado hoy en día busca algo más que un "sueldo estable", dicho incentivo, aunque sigue siendo de alta preferencia, ya pasó a segundo plano. Este estudio refleja que los mexicanos dan preferencia a un desarrollo profesional, tener un balance con la vida personal y laboral, gustan de un clima laboral sano, etc.

En complemento con lo anterior, Petit (2018) menciona que actualmente, las nuevas generaciones buscan establecerse en empresas que impulsen el desarrollo personal y profesional, por lo que un salario relativamente alto ya no garantiza un óptimo desempeño en su trabajo ni su permanencia dentro de una compañía, si el empleado llega a sentirse insatisfecho con el ambiente laboral o las oportunidades de crecimiento, no dudará en buscar buenos y mejores incentivos en otra organización.

Dentro del artículo "El sueldo no es suficiente para atraer y retener a los mejores" se observan varias conclusiones de dicho estudio, entre las que destacan que; las empresas peruanas que fueron objeto de estudio aplican mecanismos de retribuciones para retener a sus empleados, dicho mecanismo no se basa únicamente en las remuneraciones competitiva, las autoras Fuch y Sugano (2009) mencionan dichos mecanismos permiten el adecuado balance de vida personal y profesional de los empleados.

La investigación de Montoya, Portilla y Benjumea (2008) nos arroja el término "Valor percibido por los empleados" (VPE) mejor conocido como "Salario emocional", donde nos dicen que las personas trabajan cada vez más por alternativas de compensación diferentes a la económica. El VPE o Salario Emocional se ve influido positivamente por el salario, desarrollo profesional, reconocimiento en un proyecto atractivo y desafiante, variedad de actividades en el sitio de trabajo, así como desafíos en el mismo, y por los diferentes contactos profesionales que se le pueda otorgar y se verá deteriorado por factores tales como: incomodidades que pueda tener el puesto de trabajo y riesgos, definidos como inestabilidad de la empresa.

Por eso es importante que las organizaciones evolucionen dentro de la gestión administrativa al incluir modelos que integren factores motivacionales para sus empleados, siendo un punto muy importante, ya que el capital humano es quien lleva a la organización al logro de los objetivos organizacionales. El modelo mencionado es el ya conocido salario emocional o el valor percibido por el empleado, el cual, al medirlo internamente, logra identificar los focos rojos de inconformidad, lo que facilita la gestión de la motivación, lo que se traduce en una mejora continua, que, a su vez, forma empleados competentes, eficaces y eficientes, comprometidos con las metas de la organización, al mismo tiempo que la empresa refrenda su compromiso con los empleados y fortalece a la organización con un alto desempeño (Castro, Padilla y Ramírez, 2009).

En lo que respecta al dar para recibir, es normal que el trabajador aprecie las retribuciones que se le otorgan a cambio de la prestación de sus servicios, es así como aparece nuevamente el valor percibido por el empleado, resultado de la apreciación de dichas retribuciones, que, a su vez, tiene influencia sobre la satisfacción laboral, es decir la disposición psicológica de la persona hacia su trabajo (López et al., 2018). El aspecto más relevante sobre la percepción del valor percibido, es que los trabajadores tienden a preferir puestos que les permitan aplicar sus conocimientos, habilidades y capacidades, además de que le ofrezcan variedad de tareas, libertad y retroalimentación sobre lo que están haciendo.

Dentro de los incentivos del salario emocional, se encuentra la muy solicitada conciliación laboral y personal, la cual, tiene como objetivo mejorar la calidad de vida del trabajador, ya que le permite compaginar sus facetas laborales, familiares y personales. Este incentivo conocido como flexibilidad laboral, requiere la 
participación activa de la empresa, debido a que, el no poder compatibilizar la vida laboral y personal no sólo tiene repercusiones negativas en el trabajador, de igual manera, la empresa se ve afectada al aumentar los niveles de ausentismo y una baja en la productividad laboral. Por lo tanto, incluir este estimulo dentro de las retribuciones del empleado, se considera como una herramienta básica en la gestión del recurso humano, puesto que permite maximizar las potencialidades del trabajador (Belda, 2012).

Continuando con los beneficios que implica la oferta de flexibilidad, Buqueras y Cagigas (2017) señalan que el rendimiento laboral del empleado decae a partir de un determinado número de horas laboradas, lo que es totalmente natural. Los autores comentan que se trata de que el trabajo se valore por objetivos y resultados, y no de premiar la presencia, ya que esta no garantiza un buen rendimiento. Sin embargo, no se plantea la idea de trabajar menos, sino de trabajar mejor, optimizando el tiempo a través de la puntualidad, planificación, organización de la mesa de trabajo, tomar iniciativas, reducir las interrupciones durante el día, etc. Aquellas empresas que se comprometan con la excelencia en la gestión de tiempos, obtendrán una ventaja competitiva a mediano y largo plazo, reflejándose en optimización del tiempo de trabajo, racionalización y flexibilidad de horario, conciliación entre la vida personal, laboral y familiar del empleado, y sin duda aumentarán sus niveles de productividad empresarial.

Del mismo modo, coincidiendo con la opinión del párrafo anterior, Cervantes (2005) señala que el valor del empleado debe medirse a través de su rendimiento y del valor que aporta a la compañía y no sobre las horas de presencia en la oficina. Puesto que, al encontrarnos en un entorno económico y empresarial en constante cambio e incertidumbre, las empresas deben dotarse de una organización más flexible, al ser, dicha flexibilidad, un elemento clave que determinará la competitividad de la empresa y una política de doble sentido, ya que con ello se mejora la conciliación de la vida personal y laboral del empleado, así como reflejar un incremento en su responsabilidad hacia la empresa; mientras que la empresa gana efectividad, eficacia y productividad, así como mejoras en la lealtad moral y compromiso hacia la fuerza de trabajo, además de ser un instrumento de retención y atracción de talento.

Visto que, el tiempo libre y el tiempo en que se labora, son dos partes fundamentales del tiempo de la vida de un trabajador, siendo el segundo un condicionante del primero. Con la incorporación de la flexibilidad laboral se pretende disminuir el desempleo para lograr un equilibrio entre el tiempo que se dedica al trabajo, al descanso y a la superación personal (Sosa y Silva y Fuentes, 2010).

Por su parte Restrepo (2014) menciona que, según datos de la Organización Mundial de la Salud, el estrés laboral surge a raíz de horarios de trabajo estrictos e inflexibles, jornadas de trabajo largas o fuera de horario normal, horarios de trabajo imprevisibles y turnos mal concebidos. Por ello la autora aborda la flexibilidad laboral como un factor significativo dentro del salario emocional el cual debería formar parte de todo aquel programa de bienestar y responsabilidad social de la empresa, además de que refleja una excelencia en el incremento de la motivación y productividad.

Una vez puestos en perspectivas los puntos anteriores, y a modo de ejemplificar qué incentivos forman parte del salario emocional, a continuación, se enlistarán los que el autor Nicolás (2011) menciona en su investigación:

- Conciliación de vida personal y profesional a través de flexibilidad laboral, por medio de reajustes de horarios (entrada, salida y merienda), jornadas reducidas, o home office. 
- Tiempo para temas personales por medio de días libres bajo condiciones especiales o emergencias.

- Planes de carrera con oportunidades de promoción o aumento progresivo de responsabilidades y proyectos, programas de formación académica, capacitaciones e idiomas.

- Mejora de las condiciones laborales a través de buzón de sugerencias, mejor comunicación con los superiores, respeto a los horarios de salida, reconocimiento del personal y mejora en el clima organizacional.

- Ayudas personales, pago de transporte, vales para restaurantes, ticket de guardería, planes de pensiones, ayuda para gastos escolares de los hijos, etc.

- Servicios adicionales con descuento en gimnasios, clases de relajación, asesorías nutrimentales, etc.

- Actividades de voluntariado por medio de intermediarios como asociaciones civiles.

- Espacios de distracción como gimnasios privados y salas multimedia con videojuegos, billar y hasta mini golf.

- Ambiente laboral sano, inclusión, igualdad y diversidad social.

- Salud mental, trabajar el estrés laboral por medio de consultas de psicología, masajes, clases yoga y/o meditación.

Como se observó, las organizaciones actuales deben rediseñar sus estrategias para poder adaptarse a los mercados actuales, con el fin de responder a las exigencias y necesidades de los clientes, buscar mayor productividad y competitividad y lograr comprometer a sus empleados con el cumplimiento de los objetivos organizacionales. (Puerto, 2010, citado por Quintero y Betancur, 2018).

Los logros mencionados en el párrafo anterior se encuentran apoyados de teorías administrativas que parten del siglo XX, que al modernizarse aparecen como un nuevo constructo que aporta a la calidad de la empresa, al clima organizacional, la motivación y la retención de los colaboradores (Quintero y Betancur, 2018).

Por consiguiente, se dice que, cualquier empresa que desee aumentar el factor productividad, deberá tener presente la implementación del salario emocional hacia sus trabajadores. No hay que olvidar que el mayor activo de una empresa es su capital humano. Por ello es importante que la empresa motive a sus trabajadores por medio de paquetes y modelos de compensación (Zubiri, 2013). Así mismo, el autor menciona que cuando el mercado de trabajo no presenta crisis y existe bonanza laboral, la retribución económica deja de ser el elemento principal al momento de seleccionar un puesto. Entre las principales razones tanto de permanencia como de abandono dentro de las organizaciones destacan los aspectos de tipo humanitario; los profesionistas cada vez valoran más la flexibilidad, la autonomía, los programas de apoyo, la formación y las medidas de conciliación de la vida personal y laboral; es lo que se ha venido llamando, salario emocional.

Dentro de su investigación, los autores Gil, Puerta, Quintero y Ruiz-Santacruz (2019) muestran a través de un análisis empírico que toda aquella empresa que desee mayores ventas, mayor presencia en el mercado y aumentar su cartera de clientes, es decir una mayor productividad, deberá en primera instancia gestionar una estrategia que logre la satisfacción del empleado. Fue por medio de un modelo 
de salario emocional que se logró encontrar la relación entre satisfacción, empleo y productividad del empleado, al mismo tiempo de que se logra formar empresas saludables tanto en el entorno interno como externo.

Para Marshall (2016) el establecer remuneraciones o incentivos salariales basados en el desempeño, es una manera de generar un estímulo en el crecimiento de la productividad, que se refleja de igual manera en el rendimiento de los individuos o de distintas unidades o equipos de trabajo al interior de las empresas.

Por su parte, Páez (2014) por medio de un plan de salario emocional, (el cual fundamentó con la teoría de Frederick Herzberg), buscó reducir los índices de rotación, concluyendo que efectivamente, las estrategias y factores aplicados mediante el modelo de salario emocional afectaron de manera positiva a la reducción en los índices de rotación de personal, concluyendo que las estrategias derivadas de la implementación de dicho plan, afectaron de manera positiva a la retención del talento.

Un factor determinante dentro de la gestión del recurso humano es la satisfacción laboral y personal, al constatarse que aquellos trabajadores satisfechos en ambos rubros son, generalmente, los más productivos (Martínez, 2007, citado por Sánchez-Sellero, Sánchez-Sellero, Cruz-González y Sánchez-Sellero, 2014). Por consiguiente, la satisfacción debe analizarse dentro del comportamiento organizacional debido a su relación con la calidad en el desempeño, rendimiento, etc. Por otro lado, la motivación laboral son aquellos estímulos que posee el trabajador, lo que lo conduce a actuar de la mejor o peor manera en su área de trabajo.

Respecto a las retribuciones emocionales, los autores Soler y Moreno (2013, p. 1032) comentan: "La búsqueda bibliográfica permite constatar que la política retributiva de compensar al trabajador con algo más que dinero es una práctica que ha ido aumentando dentro de las empresas y que los trabajadores empiezan a valorar muy positivamente".

Sin embargo, como consecuencia del escaso uso de las retribuciones emocionales, la dificultad de su aplicación, el costo o las posibles desigualdades a la hora de utilizarlas, las empresas se encuentran renuentes a su aplicación, a pesar de que, tienen resultados positivos tanto para el trabajador como para la organización (Soler y Moreno, 2013).

En solitario y por medio de su tesis doctoral, Soler (2016) menciona que el salario emocional está dividido en dos categorías, la primera, es la retribución intangible-no económica, la segunda son los beneficios sociales. Ambas, son parte importante para la mejora del trabajador en aspectos personales y profesionales. Un dato a resaltar, que se menciona dentro de dicha investigación, es que las empresas españolas aplican dichas medidas para beneficiar el equilibrio de la vida personal y profesional de los trabajadores de aquel país.

Por otra parte, para Torres-Flórez (2019) es muy común encontrar en las organizaciones una comunicación deficiente respecto a las compensaciones que se ofrecen a los colaboradores. Es muy común ver que el trabajador sólo percibe un salario neto como única compensación, por ello es importante saber que se incluye dentro de cada concepto: 
Tabla 2. Incentivos

Término

Compensaciones

Salario

Aumentos

Incentivos

Beneficios sociales

Salario emocional
Descripción

Las compensaciones no es solamente hablar de un sueldo, aquí es importante informar al trabajador cuales son las estrategias que se tienen sobre compensaciones, y la importancia que tienen los aspectos organizacionales para desarrollarla.

La organización debe aplicar un método salarial que le permita establecer matemáticamente el valor del dinero, además de analizar variables como puesto de trabajo, el costo de vida en la región y la competitividad del sector.

Al pasar de los años el salario pierde poder adquisitivo, por ello es que las empresas realizan un aumento anual salarial a quienes perciban el salario mínimo para que el trabajador mantenga su capacidad de compra de bienes o servicios. Sin embargo, la empresa no está obligada a realizar el aumento a quienes perciban más del salario mínimo.

Estos son formas temporales de motivar al empleado, los incentivos deben estar conectados al sistema de evaluación de desempeño, debido a que cada persona debe recibir incentivos relacionados a su forma de desarrollar su labor.

A diferencia de los incentivos los beneficios sociales son para todos los colaboradores sin importar su desempeño, en ello existen los legales (vacaciones, primas, aguinaldos, pensiones, etc.) y extralegales (fiesta de fin de año, actividades recreativas, días de campo, flexibilidad de horario, permiso de maternidad, etc.).

Es una nueva tendencia dentro de las organizaciones en las que principalmente se enfoca en dar al trabajador un balance entre su vida personal y laboral.

Fuente: Elaboración propia con base en Torres-Flórez (2019).

Por otro lado, dentro de los beneficios de mantener la satisfacción laboral en el empleado, producto del uso del salario emocional, es el alza del rendimiento laboral. Por un lado, se destaca la importancia que tiene el salario emocional para obtener trabajadores satisfechos, la satisfacción se traduce a una mayor productividad y sentimiento de afiliación hacia la compañía; lo que lleva a la necesidad de evolucionar en términos de "nuevas" retribuciones, crear empresas socialmente responsables y comprometidas con sus trabajadores; del mismo modo Rocco (2009) abrió campo para entender que un empleado insatisfecho le cuesta más a la empresa hablando en términos económicos, ya que además de manejar un bajo nivel de rendimiento, es más propenso a cometer errores o a ausentarse de su trabajo, siendo la empresa quien deberá pagar el costo de la insatisfacción.

Por lo tanto, un empleado satisfecho tiende a ser más productivo, creativo y comprometido, y si se puede crear un ambiente capaz de atraer, motivar y retener a aquellos que trabajan bien, los sujetos estarán más propensos a tener éxito y ser competitivos, ya que, al igual que Nicolás (2011), la autora asevera que en algún punto el dinero disminuirá su valor al cubrir en su totalidad las necesidades del empleado, es ahí cuando la empresa tiene que mantener el alto nivel de satisfacción de sus empleados, con remuneraciones 
que van más allá de las otorgadas (Rocco, 2009).

Las empresas poco a poco han ido convenciéndose de que la retención estrategia de sus empleados requiere de la misma atención que se les da a sus ventas, por ello, Barragán, Castillo, Villalpando y Guerra (2009) mencionan en su artículo que para lograr la retención es necesario que el empleado se mantenga motivado, valorado e identificado con la empresa, por lo que será menos propenso a abandonar la compañía, de lo contrario, un empleado insatisfecho impacta directamente en los resultados operativos.

Similar a la opinión anterior, Peña y Villón (2018) en su artículo analizan cómo influye la motivación en el talento humano para lograr el éxito organizacional, los autores nos dicen que el motivar a los empleados se ha convertido en un rasgo organizacional para lograr el éxito, por lo que concluyen que las necesidades de los empleados deben ser cubiertas para que el empleado trabaje motivado y se sienta parte de la organización, de lo contrario y en convergencia con el párrafo anterior, el empleado desmotivado repercutirá de manera negativa en el éxito de la organización.

Otro alcance respecto a la satisfacción y su relación con el sueldo económico, es el de los autores Borra y Gómez (2012) quienes investigaron la satisfacción laboral en conjunto con el salario, el cual compensa de algún modo las condiciones no monetarias del trabajo, ya que según la teoría microeconómica convencional dice que un nivel alto de salario está asociado directamente con los altos niveles de satisfacción laboral, por lo que se concluyó a través de su investigación que: "El salario es exógeno en la ecuación de satisfacción laboral y tiene un efecto significativamente positivo sobre la satisfacción laboral y, segundo, existe un trade-off entre el salario y las facetas (positivas o negativas) del empleo" (p. 28).

Es decir, que el empleado valorará el sueldo que percibe a cambio de sus servicios, siempre y cuando venga de la mano de alguna formación continua que sea patrocinada por la empresa, y, además, que se dé el poder conciliar la vida profesional y personal, de lo contrario, la negatividad laboral se hará presente; el trabajador no valora el realizar horas extra sin ningún tipo de remuneración.

La política de retribuciones usada popularmente por las administraciones organizacionales se ha basado exclusivamente en la contraprestación económica. El sueldo ha sido desde un inicio el principal factor motivador y casi el único premio a cambio del esfuerzo del trabajador. Sin embargo, Padilla (2013) en su artículo, menciona que desde el inicio de las primeras décadas del siglo XX, se ha demostrado que el ser humano se mueve a través de diferentes estímulos, que van más allá de incentivos monetarios; es lo que se ha venido llamando "salario emocional", el cual no sólo premia el rendimiento, sino también la conducta, competencias, la formación y definitivamente, el desempeño del trabajador.

No obstante, son pocas las evidencias que demuestren que ofrecer un mayor sueldo al empleado sea el factor principal para la retención o atracción de talento, como se ha visto a lo largo de este artículo. La mayoría de los estudios muestran que los factores monetarios no es la razón más importante para que una persona acepte o deje un trabajo. Es por ello que surge la duda, de si será conveniente que el departamento de RH ponga sus ojos en una estrategia de retención basada en el sueldo económico (Gonzales, 2009). El autor menciona que es bien sabido que el trabajo es el principal medio para la obtención de ingresos, y cubrir las necesidades básicas del mismo, pero no es la única razón por la que el trabajador permanece en el trabajo, sin embargo, al mismo tiempo, comenta que las empresas pueden poner en práctica las medidas que consideren necesarias para la retención de talento, pero, al final del día, la disposición de quedarse obedecerá a la decisión libre y autónoma del empleado. 
De acuerdo con Spector (2002, citado por Barragán et al., 2009) la satisfacción laboral es tan importante que produce fuertes vínculos con los empleados y las organizaciones, desde el desempeño laboral hasta la salud y la longevidad. Así mismo, Barragán et al. (2009) citando ahora a Blum (1990), ponen en perspectiva que la satisfacción en el trabajo es el resultado de las actitudes del empleado, las cuales se derivan y tienen relación con factores específicos como el sueldo, la supervisión, la constancia en el trabajo, las condiciones laborales, asensos, reconocimiento, retroalimentaciones y evaluaciones justas, relaciones sociales en el trabajo, la resolución oportuna de quejas, el trato justo por parte del patrón y demás conceptos similares.

Un incentivo considerado dentro del salario emocional que no se ha mencionado aún, siendo este el más solicitado por el empleado, es la capacitación. Cota y Rivera (2017) mencionan por medio de su artículo, que, si una organización desea mejorar sus procesos y ser más competitiva, debe invertir en sus colaboradores, es decir, capacitarlo. Los beneficios de capacitar al empleado van desde formar líderes, mejorar la comunicación entre el personal y conduce a la rentabilidad. Por otro lado, un beneficio social de la capacitación es el valor agregado que el empleado obtiene, sus conocimientos, destrezas y aptitudes se perfeccionan al momento de trabajar, además de repercutir en su vida personal. En pocas palabras, las promociones, traslados y actividades de capacitación son un importante factor de motivación y retención de personal.

De acuerdo con los autores Parra-Penagos y Rodríguez-Fonseca (2015), afirman que la capacitación es una estrategia que las organizaciones deben tomar de manera sistemática para crear un desarrollo y sostenimiento de la empresa a través de los conocimientos del empleado. Dentro del artículo, se menciona que la formación profesional ayuda a mejorar las habilidades y conocimientos de los trabajadores, lo que beneficia a la organización en cuanto a eficiencia, calidad y servicio al cliente.

Y para concluir con el tema de capacitación, Bermúdez (2015) expone que dentro de un mundo cada vez más globalizado, las empresas altamente competitivas son aquellas que mantienen a su personal motivado, y que la mejor manera de lograrlo, es por medio del grado de conocimiento y capacitación que se le ofrezca, por ello, la empresa debe tener apertura para capacitarlo.

Ahora, ¿Cuál es la manera más simple, segura y directa de conseguir que el empleado haga lo que se le pide?, muchos gerentes aún aplican el método "KITA" (Kick in the ass, por sus siglas en inglés) literalmente traducido como ¡Dele una patada!, el cual consiste en tres modalidades, la primera es el de utilizar la fuerza (literalmente), la segunda consiste en utilizar ataques psicológicos para logra vulnerabilidad, y la tercera ofrecer recompensas a cambio del trabajo, muchos gerentes, dirán que es el método más seguro y con menos rodeos. Ahora bien, ¿Por qué KITA no es una motivación?, se dice que el empleado no debería necesitar una estimulación externa, debería querer hacerlo (Herzberg, 1969).

Por ello, es que se revisaron algunas prácticas KITA que al utilizarlas de manera positiva logrará un desarrollo adecuado en la motivación, entre ellas se destacan las reducciones en las horas de trabajo, la motivación a través de un aumento de sueldo, beneficios extra-salariales, capacitaciones, comunicación de dos vías y participación en el trabajo, sin embargo, cabe destacar que este método sólo funciona a corto plazo, ya que el costo de este programa aumenta continuamente (Herzberg, 1969).

La motivación se puede generar desde: ampliar las responsabilidades del empleado, hacerlos participes de las decisiones o del funcionamiento de la empresa, mantener la motivación en la participación en cuanto ideas nuevas, generar desafíos laborales, recompensar de manera justa, equitativa sin descuidar 
la motivación salarial; todo ello son las conclusiones del artículo publicado por los autores Garcés, Londoño, Méndez y Martínez (2015)

Una vez considerados estos antecedentes, se consolida cada vez más la idea de que los elementos que proporciona el salario emocional, al ser debidamente gestionados, lograrán que el trabajador desarrolle un sentido de pertenencia más arraigado con la organización, provocando su permanencia en la misma y finalmente un bajo índice de rotación de personal.

No obstante, y pese a que la literatura consultada demuestra que el salario emocional trae consigo múltiples beneficios tanto para el empleado como para la empresa, no todo son ventajas, como bien lo explican Daza y Torres (2015). Los autores mencionan que, por ejemplo, el teletrabajo o el uso de horarios flexibles no maneja un sueldo igual de atractivo que el de una jornada de ocho horas en la oficina, y sus remuneraciones son inferiores a la media, además, el teletrabajo requiere más disciplina; otra desventaja es que el empleado puede llegar a confundir el incentivo emocional como una obligación, siendo que el salario emocional no es obligatorio, ni por ley, y por último, un empleado que anteriormente haya disfrutado de los beneficios del salario emocional y recién sea contratado por una empresa donde no lo ofrezcan puede ser un problema, el empleado puede exigirlo para su permanencia o bien rechazará el puesto.

A modo de cierre, se abordará una pregunta de interés personal, ¿Por qué las empresas traducen la implementación del salario emocional como gasto?, las organizaciones muchas veces olvidan que el trabajador es su principal activo y por ende su principal cliente interno, es por eso que cualquier beneficio extra que pueda obtener por parte de la empresa debe verse como una inversión más que como un gasto; aunque, Enciso (2017), menciona que, pequeños cambios que se traducen como integradores del salario emocional, los cuales no requieren gran inversión monetaria, sino compromiso y monitoreo, alineados con los objetivos y estrategias organizacionales de la compañía, pueden generar un valor agregado a la relación empleado-empleador, el compromiso de la alta gerencia y de los líderes de cada proceso, es vital en la de consecución de objetivos que inspiran a trabajar en equipo y a ser más competitivos en el entorno.

\section{Conclusiones}

En este artículo se ha demostrado a través de la revisión documental los beneficios del uso del salario emocional. Una vez finalizado el presente estudio se arrojan las siguientes conclusiones. Las investigaciones previas publicadas en la literatura analizada nos dicen que hoy en día el uso del salario emocional dentro de las organizaciones es considerado un elemento importante para el incremento del rendimiento, motivación y productividad laboral del empleado, del mismo modo, elementos como satisfacción, motivación y fidelización se ven impactados de manera positiva, ya que, a pesar de contar con un empleo "estable" y bien remunerado, el trabajador buscará un lugar donde pueda encontrar un balance entre su sueldo y beneficios emocionales.

Los beneficios que trae consigo el salario emocional también aplica para quienes están en la búsqueda de su primer empleo, es decir, dependiendo del nivel de salario emocional proporcionado por la empresa, el empleado decidirá si está dispuesto a aceptar o no el trabajo. Sin dejar a un lado a la organización, quien es el prestador de dicho beneficio, el uso del salario emocional beneficiará a la empresa en cuanto a eficiencia, eficacia, productividad y competitividad, además de lograr la fidelización del talento humano 
y reforzar los objetivos de la organización. Por otra parte, una empresa que se preocupa por el bienestar de sus empleados refleja un clima laboral sano, por consecuencia hará de la organización un lugar más atractivo tanto en la etapa de reclutamiento y la permanencia del empleado.

No todo es ventajas y beneficios dentro del uso del salario emocional, hoy en día debido a factores financieros y económicos hay empresas que no pueden realizar el gasto que implica el incorporar remuneraciones emocionales para sus empleados, además de que la implementación de este no garantiza al 100\% la fidelidad y permanencia del empleado a la organización. El salario emocional puede llegar a confundir al empleado y hacerlo creer que es un beneficio obligatorio por parte del patrón.

\section{Bibliografía}

Aranguren, B. (2020). El salario emocional como estímulo para la retención de talento. ORH.

Asociación de Internet MX (2018). Informe 2018. Recuperado de: https://www.asociaciondeinternet.mx/. Augusto, T., Campagnolli, D., Canuto, T. y Graziano, G. (2018). Emotional Salary As A Strategy To Retain Talents. IOSR Journal Of Humanities And Social Science, 74-80.

Barragán, J. N., Castillo, J., Villalpando, P. y Guerra, P. (2009). La retención de empleados eficientes: importancia estratégica de la fidelización de los empleados. Innovaciones de negocios, 6(11), 33-43.

Belda, P. R. (2012). Satisfacción con la conciliación laboral y familiar de los asalariados en España. Revista de Economía Laboral, 9(1), 30-45.

Bermúdez, L. A. (2015). Capacitación: una herramienta de fortalecimiento de las pymes. InterSedes, 16(33), 01-25.

Borra, C. y Gómez, F. (2012). Satisfacción laboral y salario: ¿compensa la renta laboral las condiciones no monetarias del trabajo? Revista de Economía Aplicada, 20(60), 25-51.

Buqueras, I. y Cagigas, J. (2017). Dejemos de perder el tiempo: Los beneficios de optimizar los horarios. Lid Editorial.

Castro, M. G., Padilla, J. M. y Ramírez, S. M. (2009). Grado de satisfacción laboral y condiciones de trabajo: una exploración cualitativa. Enseñanza e investigación en Psicología, 14(1), 105-118.

Cervantes, M. (2005). Las ventajas de la empresa flexible. UCJC Business and Society Review (antes conocida como Universia Business Review), 5.

Cota, J. A. y Rivera, J. L. (2017). La capacitación como herramienta efectiva para mejorar el desempeño de los empleados de una cadena de zapaterías de ciudad Obregón, Sonora. Técnica Administrativa, 16(2), 0.

Daza, G., y Torres, J. D. (2015). Salario emocional como estrategia para la perdurabilidad en los colaboradores de mejor rendimiento en Industria de Alimentos Daza S.A.S. Recuperado de: https://ciencia.lasalle. edu.co/administracion_de_empresas/576.

Enciso, K. A. (2017). Influencia del salario emocional en la calidad de vida y productividad en las organizaciones actuales. Tesis de maestría. Repositorio Institucional UMNG.

Fuchs, R. M. y Sato, S. S. (2009). El sueldo no es suficiente para atraer y retener a los mejores. Journal of Business, 1(2), 91-106.

Garcés, C., Londoño, I., Méndez, K. y Martínez, J. (2015). Retención de empleados, una estrategia para el éxito de las organizaciones. Revista Fundación Universitaria Luis Amigó, 3(1), 108-115. 
Gil, V. D., Puerta, I., Quintero, L. F. y Ruiz-Santacruz, J. S. (2019). Development of an emotional salary model: a case of application. Indian Journal of Science and Technology, 12(42), 1-18. DOI: 10.17485/ ijst/2019/v12i42/146964.

Gonzales, D. R. (2009). Estrategias de retención del personal Una reflexión sobre su efectividad y alcances. Revista Universidad EAFIT, 45(156), 45-72.

González, F. y De Avice, A. D.(2017). Qué es y cómo se paga el salario emocional. Revista de negocios del IEEM, 80.

Granados, I. (2011). Calidad de vida laboral: historia, dimensiones y beneficios. Revista de investigación en Psicología, 14(2), 209-221.

Herzberg, F. (1969). Una vez más: ¿cómo motiva a sus empleados? Harvard Business Review. Reimpresión 2003. Recuperado de: https://dialnet.unirioja.es/servlet/articulo?codigo=1431362.

López, C., Chávez, A., Peña, M. y Guevara, J. (2018). Valor percibido por el empleado sobre su satisfacción laboral. Revista Internacional Administración y Finanzas, 11(3), 95-105.

Marshall, A. (2016). La relación salarios-productividad: incentivos salariales en los convenios colectivos industriales. Trabajo y sociedad, 26, 5-22.

Martín, S. (2017). La Satisfacción Laboral en relación con el Salario Emocional. Tesis de licenciatura. Repositorio institucional RIULL.

Montoya, L. A., Portilla, L. M., y Benjumea, J. C. C. (2008). Compensación y beneficios salariales; atracción y retención de trabajadores. Scientia et technica, 14(39), 265-268.

Nicolás, M. A. (2011). El salario emocional. Revista agropecuaria y ganadera, 692-693. Recuperado de: https://dialnet.unirioja.es/servlet/articulo? codigo=3798882.

Padilla, P. (2013). La gestión del reconocimiento en la administración pública. Estudios financieros. Revista de Trabajo y Seguridad Social: Comentarios, casos prácticos: recursos humanos, 364, 189-206.

Páez, J. R. (2014). Disminución de los Índices de Rotación de Personal en la empresa Panatlantic Logistics S.A mediante la aplicación de un Plan de Salario Emocional. Tesis de licenciatura. Quito: UCE.

Parra-Penagos, C. y Rodríguez-Fonseca, F. (2016). La capacitación y su efecto en la calidad dentro de las organizaciones. Revista de investigación, desarrollo e innovación, 6(2), 131-143. DOI: http://dx.doi. org/10.19053/20278306.4602.

Peña, H. C. y Villón, S. (2018). Motivación Laboral. Elemento Fundamental en el Éxito Organizacional. Revista Scientific, 3(7), 177-192. DOI: https://doi.org/10.29394/Scientific.issn.2542-2987.2018.3.7.9.177192.

Petit, H. (2019). Empresas comprometidas con el salario emocional de sus colaboradores en el sector minero de Colombia. Consensus (Santiago)-Revista interdiscipliaria de investigación, 3(1), 03-23.

Puyal, F. G. (2006). El salario emocional, clave para reducir el estrés. Gestión práctica de riesgos laborales: Integración y desarrollo de la gestión de la prevención, 33, 44-47.

Quintero, L. F. y Betancur, J. D. (2018). Modelo de salario emocional para la fidelización de los colaboradores en la búsqueda de una organización competitiva. Espacios, 39(41), 8-13.

Restrepo, P. L. (2014). La flexibilidad laboral y el salario emocional. Aglala, 5(1), 34-68.

Rocco, M. T. (2009). Satisfacción Laboral y Salario Emocional: Una aproximación teórica. Tesis de licenciatura. Repositorio académico de la Universidad de Chile. 
Sánchez-Sellero, M. C., Sánchez-Sellero, P., Cruz-González, M. M. y Sánchez-Sellero, F. J. (2014). Características organizacionais da satisfação no trabalho na Espanha. Revista de Administração de Empresas, 54(5), 537-547.

Soler, G. (2016). El salario emocional para el equilibrio de la vida personal y profesional en los centros universitarios. Un estudio de FUNITEC (URL), EPSEB (UPC) y ESPEVG (UPC). Tesis doctoral. Universitat Ramon Llull.

Soler, G. y Moreno, C. (2013). Inversión en la retribución tangible para la conciliación laboral. Intangible Capital, 9(4), 1021-1041. DOI: http://dx.doi.org/10.3926/ic.444.

Sosa y Silva, Y. y Fuentes, G. (2010). Menos tiempo de trabajo, mejor calidad de vida. Ciencia $y$ Cultura, 139-158. Recuperado de: http://repositorio.lasalle.mx/bitstream/handle/lasalle/467/ N\%C3\%BAm.14_P.139-158.pdf?sequence=1.

Torres-Flórez, D. (2019). Estrategia de compensaciones como herramienta de satisfacción laboral. Revista GEON (Gestión, Organizaciones y Negocios), 6(2), 4-9.

Zubiri, F. (2013). Satisfacción y motivación profesional. Anales del sistema sanitario de Navarra, 36(2), 193-196. 